\title{
Acoustic and Electrical Properties of Piezoelectric Materials for High-Temperature Sensing Applications ${ }^{\dagger}$
}

\author{
Ward L. Johnson \\ National Institute of Standards and Technology, 325 Broadway St., Boulder, CO 80305, USA. \\ wjohnson@boulder.nist.gov
}

\begin{abstract}
:
Sensing applications at temperatures above $500{ }^{\circ} \mathrm{C}$ have motivated research on a number of innovative single-crystalline piezoelectric materials that are compositionally and structurally stable at these temperatures. These materials include ordered and disordered crystals in the langasite family, gallium orthophosphate, rare-earth calcium oxyborates, and crystals with the structure of melilite. In this review, measurements and analysis of the properties of these materials relevant to the operation of piezoelectric acoustic sensors at elevated temperatures are summarized, with a primary focus on acoustic loss arising from temperature-dependent electrical conductivity.
\end{abstract}

Key words: Acoustic sensors, calcium aluminate silicate, CTGS, gallium orthophosphate, gallium phosphate, high temperatures, langasite, langatate, piezoelectric materials, rare-earth oxyborates.

\section{Introduction}

A variety of sensing applications in the temperature range above $500{ }^{\circ} \mathrm{C}$ are driving research on the growth and characterization of innovative piezoelectric single crystals for resonant bulk-acoustic-wave (BAW) and surface-acoustic-wave (SAW) devices that can operate in this temperature range. Proposed applications include monitoring of temperature, pressure, acceleration, and vibration in internal combustion engines, aerospace propulsion systems, power plants, and solid oxide fuel cells [1-6]. The objectives of such monitoring include optimization of performance and efficiency and monitoring of structural health. High-temperature piezoelectric crystals also offer extended capabilities for monitoring small mass changes for thin-film deposition systems [7-9], space applications [10], and thermogravimetric analysis [11,12].

Piezoelectric crystals that maintain nominal compositional and structural stability above 500 ${ }^{\circ} \mathrm{C}$ include ordered and disordered crystals with the structure of langasite $\left(\mathrm{La}_{3} \mathrm{Ga}_{5} \mathrm{SiO}_{14}\right.$, "LGS"), gallium orthophosphate $\left(\mathrm{GaPO}_{4}\right)$, rare-earth calcium oxyborates $\left(R e C a_{4} \mathrm{O}\left(\mathrm{BO}_{3}\right)_{3}\right.$, where "Re" denotes a rare-earth element), and crystals with the structure of melilite.

\footnotetext{
† This manuscript is a contribution of the National Institute of Standards and Technology and is not subject to copyright in the United States.
}

Since the first synthesis of langasite in 1980 , materials in this family, primarily langasite and langatate $\left(\mathrm{La}_{3} \mathrm{Ga}_{5.5} \mathrm{Ta}_{0.5} \mathrm{O}_{14}\right.$, "LGT"), have been a dominant focus of research and commercialization of innovative piezoelectric materials for high-temperature applications [1, 13-15]. These crystals in the trigonal P321 crystal class (similar to the $P 3_{1} 21$ class of quartz) [16] can be grown from the melt by the Czochralski technique. LGS and LGT have partially disordered crystal structures, with more than one atomic species randomly occupying equivalent lattice sites, and this disorder limits the electrical resistivity and associated acoustic quality factor $Q$ at elevated temperatures [14]. Numerous fully ordered crystals in the langasite family have also been identified [17-20], and two such materials, $\mathrm{Ca}_{3} \mathrm{TaGa}_{3} \mathrm{~S}_{\mathrm{i} 2} \mathrm{O}_{14}$ (CTGS) and $\mathrm{Ca}_{3} \mathrm{NbGa}_{3} \mathrm{Si}_{2} \mathrm{O}_{14}$ (CNGS), are now commercially available.

The ability to grow LGS-type crystals with the Czochralski method is a great advantage relative to the slow hydrothermal process required for synthetic quartz. On the other hand, the cost of gallium in these crystals is a limiting factor with respect to the ultimate minimal cost of crystals and the associated marketable range of applications at low or moderate temperatures (where quartz is competitive). This situation has provided part of the motivation for research on innovative piezoelectrics with other crystal structures. It has also led to exploration of the substitution of other elements for some or all of the gallium in 
LGS-type crystals. This research recently has included the growth and characterization of $\mathrm{Ca}_{3} \mathrm{TaAl}_{3} \mathrm{Si}_{2} \mathrm{O}_{14}$ and $\mathrm{CaTa}\left(\mathrm{Al}_{0.9} \mathrm{Ga}_{0.1}\right)_{3} \mathrm{Si}_{2} \mathrm{O}_{14}$ (CTAS and CTAGS) [20]. CTAS crystal growth is reported to involve substantial challenges that are overcome by the partial substitution of $\mathrm{Ga}$ for $\mathrm{Al}$ in CTAGS. However, this partial substitution makes CTAGS disordered and may result in some degradation of high-temperature resistivity.

$\mathrm{GaPO}_{4}$, which belongs to the trigonal $P 3_{1} 21$ crystal class, has also been a focus of substantial research and commercialization for high-temperature applications. It has the attractive characteristics of relatively low electrical conductivity and low temperature dependence of resonant frequencies over broad temperature ranges [14, 21-23]. However, commonly cited obstacles to widespread application of $\mathrm{GaPO}_{4}$ resonators include relatively high cost and limited availability, which are partly associated with the difficult and time-intensive hydrothermal growth process.

Research on rare-earth calcium oxyborates for high-temperature applications has been recently reviewed by $\mathrm{Yu}$ et al. [24]. This research includes crystals with rare-earth elements Er, Y, Gd, La, Nd, Pr, and Sm. The crystals have monoclinic symmetry and are grown by the Czochralski technique. Of the samples studied thus far, $\mathrm{YCa} \mathrm{Ca}_{4} \mathrm{O}\left(\mathrm{BO}_{3}\right)_{3}$ (YCOB) and $\mathrm{ErCa}_{4} \mathrm{O}\left(\mathrm{BO}_{3}\right)_{3}(\mathrm{ErCOB})$ stand out as having exceptionally high resistivities at elevated temperatures [24].

Research on crystals with melilite structure for high-temperature acoustic applications has been published only within the last couple of years [25-28], although some of these crystals, such as calcium aluminate silicate $\left(\mathrm{Ca}_{2} \mathrm{Al}_{2} \mathrm{SiO}_{7}\right)$, have been a focus of research for other applications over a longer period of time [29]. These crystals have tetragonal $\left(P 42_{1} m\right)$ symmetry and are grown by the Czochralski technique. Relative advantages for sensing applications include the low cost of starting materials for some of these compounds.

The upper temperature limits of piezoelectricity of LGS-type crystals are determined by their melting points, which are in the range of 1350 ${ }^{\circ} \mathrm{C}$ to $1470{ }^{\circ} \mathrm{C}$ [30]. The temperature limit of piezoelectricity of $\mathrm{GaPO}_{4}$ is determined by a structural phase transition at $970{ }^{\circ} \mathrm{C}$ [31]. Melting temperatures of the rare-earth oxyborates are in the range of $1400{ }^{\circ} \mathrm{C}$ to 1520 ${ }^{\circ} \mathrm{C}$ [24], and those of the melilite-structure crystals are $1550{ }^{\circ} \mathrm{C}$ to $1650{ }^{\circ} \mathrm{C}$ [25]. Practical operation of acoustic sensors is limited to temperatures somewhat below these ultimate limits because of increasing conductivity and atmosphere-dependent compositional instability at elevated temperatures.

The remainder of this report is focused on comparisons of published electrical and acoustic characteristics of LGS, LGT, CTGS, $\mathrm{GaPO}_{4}, \quad \mathrm{YCa}_{4} \mathrm{O}\left(\mathrm{BO}_{3}\right)_{3}, \quad \mathrm{GdCa} \mathrm{O}_{4}\left(\mathrm{BO}_{3}\right)_{3}$, and $\mathrm{Ca}_{2} \mathrm{Al}_{2} \mathrm{SiO}_{7}$ at high temperatures. These materials are representative of the types of crystals described above for which hightemperature data are available.

\section{Electromechanical Coupling Efficiency}

The efficiency of coupling of applied electric fields and mechanical displacements in a piezoelectric crystal can be expressed in terms of the dimensionless electromechanical coupling factor $k_{i j}$, which is a function of the effective piezoelectric constant, elastic constant, and dielectric permittivity for a specified electric field direction and strain symmetry [32]. The subscripts $i$ and $j$ on $k$ indicate, respectively, the direction of the electric field and the strain component (in reduced-index notation).

For $\mathrm{Y}$-cut quartz and the other trigonal crystals LGS, LGT, $\mathrm{GaPO}_{4}$, and CTGS, the thicknessshear electromechanical coupling factor $k_{26}$ is given by [32]

$$
k_{26}^{2}=\frac{e_{26}^{2}}{C_{66}^{E} \epsilon_{22}^{S}}=\frac{e_{26}^{2}}{C_{66}^{E} \epsilon_{11}^{S}},
$$

where $e_{26}, \epsilon_{22}^{S}$, and $C_{66}^{E}$ are, respectively, the corresponding piezoelectric constant, dielectric permittivity at constant strain, and elastic constant at constant electric field. Typical values of $k_{26}$ reported for $\mathrm{LGS}, \mathrm{GaPO}_{4}$, and CTGS at ambient temperatures are 0.13 [33], 0.19 [34], and 0.15, respectively [35]. The coupling factors $k_{26}$ for thickness-shear resonators of $\mathrm{YCa} \mathrm{O}_{4} \mathrm{O}\left(\mathrm{BO}_{3}\right)_{3}$ and $\mathrm{GdCa} \mathrm{O}_{4} \mathrm{O}\left(\mathrm{BO}_{3}\right)_{3}$ are reported to be 0.19 and 0.25 , respectively $[24,36]$. All of these coupling factors are substantially greater than the value of 0.06 for Y-cut quartz [34]. Coupling factors for thickness-shear resonators of $\mathrm{Ca}_{2} \mathrm{Al}_{2} \mathrm{SiO}_{7}$ have apparently not been published. However, values of $k_{31}$ for transverse excitation of extensional modes of this material are reported to be 0.17 [27].

Limited information has been published on the temperature dependence of coupling factors of these various crystals. Yu et al. [35] reported an approximate $10 \%$ drop in $k_{26}$ of CTGS on heating from ambient temperature to $700{ }^{\circ} \mathrm{C}$. For $\mathrm{GdCa}_{4} \mathrm{O}\left(\mathrm{BO}_{3}\right)_{3}$, Zhang et al. [36] found that $k_{26}$ dropped less than $5 \%$ on heating to 1000 ${ }^{\circ} \mathrm{C}$, and $\mathrm{Yu}$ et al. [24] found a greater drop of 
approximately $15 \%$ over the same range. $\mathrm{YCa}{ }_{4} \mathrm{O}\left(\mathrm{BO}_{3}\right)$ is reported to have a slight increase in $k_{26}$ upon heating to $1000^{\circ} \mathrm{C}$ [24].

\section{Electrical Conductivity}

Increased electrical conductivity of piezoelectric materials at elevated temperatures reduces the signal strength and resolution of piezoelectric sensors. As described in the next section, electrical conductivity is directly linked, through piezoelectric coupling, to acoustic loss and associated frequency resolution.

Figure 1 shows a sampling of published measurements of electrical conductivity $\sigma$ of $Y$ cut LGS [37], LGT [38], CTGS [37], $\mathrm{GaPO}_{4}$ [14], $\mathrm{Ca}_{2} \mathrm{Al}_{2} \mathrm{SiO}_{7}$ [26], and $\mathrm{YCa}_{4} \mathrm{O}\left(\mathrm{BO}_{3}\right)_{3}$ [39]. Measurements on ( $\mathrm{YXt})-33^{\circ}$ cut $\mathrm{GdCa}_{4} \mathrm{O}\left(\mathrm{BO}_{3}\right)_{3}$ [36] are also included in this figure. LGS and LGT have the highest conductivities of those plotted in Fig. 1, and this is consistent with the fact that these crystals are partially disordered. The conductivities of CTGS, $\mathrm{GaPO}_{4}$, and $\mathrm{Ca}_{2} \mathrm{Al}_{2} \mathrm{SiO}_{7}$ are comparable in magnitude over the measured ranges. The values reported for $\mathrm{YCa} \mathrm{O}_{4} \mathrm{O}\left(\mathrm{BO}_{3}\right)_{3}$ and $\mathrm{GdCa} \mathrm{O}_{4} \mathrm{O}\left(\mathrm{BO}_{3}\right)_{3}$ are the lowest of those in Fig. 1, although their greater slopes (and corresponding activation energies) lead to the conductivities of these crystals approaching those of $\mathrm{GaPO}_{4}, \mathrm{CTGS}$, and $\mathrm{Ca}_{2} \mathrm{Al}_{2} \mathrm{SiO}_{7}$ at the highest temperatures.

Considering the early stage of research on these crystals and potential variations in crystal composition and defects, the data shown in Fig. 1 provide only a tentative indication of the conductivities that can eventually be achieved. One should note, for example, that other reported values of $\sigma$ of $Y$-cut CTGS at a given temperature differ from each other by more than an order of magnitude [38,39].

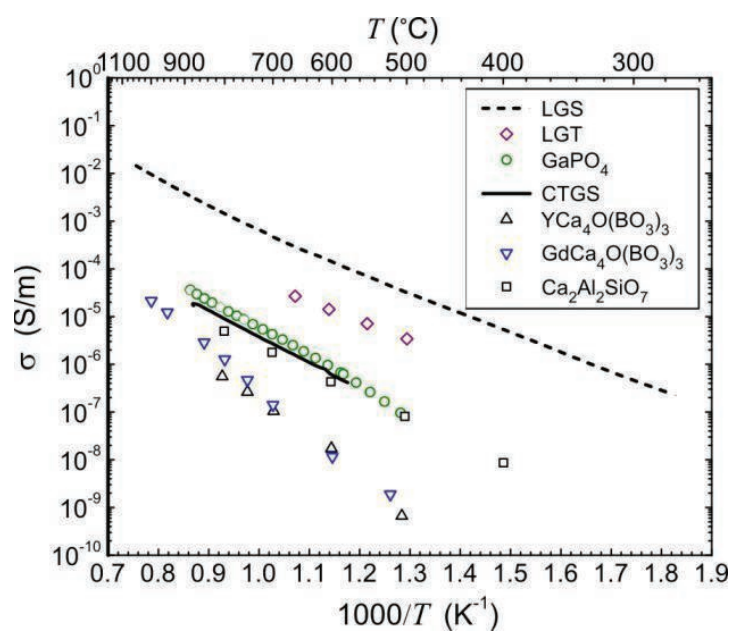

Fig.1. Temperature dependence of electrical conductivities of $Y$-cut LGS [37], LGT [38], $\mathrm{GaPO}_{4}$ [14], CTGS [37], $\mathrm{YCa}_{4} \mathrm{O}\left(\mathrm{BO}_{3}\right)_{3}$ [39], and $\mathrm{Ca}_{2} \mathrm{Al}_{2} \mathrm{SiO}_{7}$ [26], and (YXt) $-33^{\circ}$ cut $\mathrm{GdCa}{ }_{4} \mathrm{O}\left(\mathrm{BO}_{3}\right)_{3}$ [36].
The effects of point defects and doping on the conductivity of LGS have been extensively studied theoretically and experimentally [14,40 - 44]. LGS without intentional doping but with typical levels of acceptor impurities, is found to have mixed ionic-electronic conductivity, with ionic transport being dominant at high temperatures in atmospheres with medium-tohigh levels of oxygen. These characteristics have been explained by a defect model involving the formation of positively charged oxygen vacancies (the primary ionic charge carriers) in response to negatively charged acceptor impurities [40,41]. The balance between ionic, $n$-type electronic, and $p$-type electronic conduction has been explored through the introduction of acceptor and donor dopants, with the aim of establishing procedures for optimizing crystal growth. For example, $\mathrm{Nb}$ doping $(0.5 \%)$ has been found to substantially reduce conductivity up to at least $900{ }^{\circ} \mathrm{C}$ [14]. However, in this case, the conductivity was not reduced to the levels of the conductivity of CTGS or the other fully ordered crystals shown in Fig. 1.

\section{Acoustic loss}

Stress and strain of an acoustic wave in piezoelectric materials have slightly different phases because of out-of-phase piezoelectric terms in the equation of motion, in addition to any anelastic contributions from defects. Since real materials have finite conductivity, the piezoelectric coupling between mechanical and electrical effects is not instantaneous because of the non-zero dielectric relaxation time $\tau_{c}$. This significantly influences the propagation of an acoustic wave when $\tau_{c}$ is not substantially greater than the period of the wave, which occurs in piezoelectric crystals at high temperatures $[45,46]$.

The dielectric relaxation time is inversely proportional to $\sigma$ :

$$
\tau_{c}=\frac{\epsilon_{i i}^{S}}{\sigma},
$$

where $\epsilon_{i i}^{S}$ is a diagonal element of the dielectric permittivity tensor at constant strain [45]. For $Y$-cut and singly-rotated $(Y X I)$ trigonal crystals excited in the thickness-shear mode, the appropriate dielectric permittivity constant is denoted by $\epsilon_{22}^{S}\left(=\epsilon_{11}^{S}\right)$.

The acoustic phase lag arising from the piezoelectric/conductive effect leads to a contribution $Q_{c}^{-1}$ to the total damping $Q^{-1}$ that is approximately given by $[45,46]$

$$
Q_{c}^{-1}(T, \omega)=k_{i j}^{2} \frac{\omega \tau_{C}}{1+\omega^{2} \tau_{c}^{2}},
$$


where $\omega$ is the acoustic angular frequency and $T$ is the absolute temperature. Equation (3) has the form of a Debye relaxation function [47]. Temperature dependence in this equation arises through $\tau_{c}$ and, to a much lesser extent, $k_{i j}$.

If electrical transport is dominated by ionic conduction or electron hopping, $\sigma$ is given by

$$
\sigma(T)=\frac{c_{0}}{T} \exp \left(-\frac{U_{c}}{k T}\right)
$$

where $c_{0}$ and $U_{c}$ are constants [41] [48]. If transport is dominated by electron migration, this equation is modified to not include $T$ in the factor multiplying the exponential [14].

At temperatures well below the peak temperature of the Debye function (Eq. (3)), $\omega \tau_{c}$ is much greater than 1 and $Q_{c}^{-1}$ is approximately given by

$$
Q_{c}^{-1}(\omega, T) \approx \frac{k_{i j}^{2}}{\omega \tau_{c}}=\frac{k_{i j}^{2} \sigma}{\omega \epsilon_{i i}^{S}} .
$$

This is the anticipated situation at $5 \mathrm{MHz}$ for the materials considered in this review at temperatures up to at least $900{ }^{\circ} \mathrm{C}$, with the exceptions of LGS and LGT $[1,46]$. Therefore, the piezoelectric/conductive contribution to the loss leads to either $\ln \left(Q_{c}^{-1} T\right)$ or $\ln \left(Q_{c}^{-1}\right)$ being approximately linearly dependent on $1 / T$, neglecting the much weaker temperature dependence of $k_{i j}$ and $\epsilon_{i i}^{S}$.

Figure 2 shows a sampling of published measurements of $Q^{-1}$ of $Y$-cut LGS $[37,46], Y$ cut LGT [46], Y-cut $\mathrm{GaPO}_{4}$ [14], Y-cut CTGS [37], $(\mathrm{YXI})-35^{\circ}$ cut CTGS, [37] (YXt) $-30^{\circ}$ cut $\mathrm{YCa}_{4} \mathrm{O}\left(\mathrm{BO}_{3}\right)_{3} \quad[49], \quad(Y X t) \quad-33^{\circ} \quad$ cut $\mathrm{GdCa}_{4} \mathrm{O}\left(\mathrm{BO}_{3}\right)_{3}$ [36], and Z-cut quartz [50] at elevated temperatures. These measurements were performed at frequencies between $5 \mathrm{MHz}$ and $6 \mathrm{MHz}$. In none of these measurements does the temperature dependence of $Q^{-1}$ over the measured range come close to matching that expected from the data in Fig. 1 and Eqs. (4) and (5) for the piezoelectric/conductive contribution. Therefore, contributions other than piezoelectric/conductive damping dominate the total $Q^{-1}$ over a large fraction of the temperature ranges of the measurements. Note, in particular, that the exceptionally low conductivities of $\mathrm{YCa}_{4} \mathrm{O}\left(\mathrm{BO}_{3}\right)_{3}$ and $\mathrm{GdCa}_{4} \mathrm{O}\left(\mathrm{BO}_{3}\right)_{3}$ shown in Fig. 1 have not translated into $Q^{-1}$ being lower than reported values for $\mathrm{GaPO}_{4}, \mathrm{CTGS}$, or $\mathrm{Ca}_{2} \mathrm{Al}_{2} \mathrm{SiO}_{7}$ in Fig. 2. This indicates that, over most (or all) of the temperature ranges of the measurements, other sources of acoustic loss are dominant in the particular samples of $\mathrm{YCa}_{4} \mathrm{O}\left(\mathrm{BO}_{3}\right)_{3}$ and $\mathrm{GdCa}_{4} \mathrm{O}\left(\mathrm{BO}_{3}\right)_{3}$ that were studied. The two sets of measurements shown in Fig. 2 on different LGS crystals (from different crystal growers) also illustrate variations in the contributions to $Q^{-1}$ that are not related to piezoelectric/conductive damping. The values of $Q^{-1}$ in these two data sets differ by approximately an order of magnitude below $150{ }^{\circ} \mathrm{C}$ but converge towards each other at the highest overlapping temperatures (near $450{ }^{\circ} \mathrm{C}$ ).

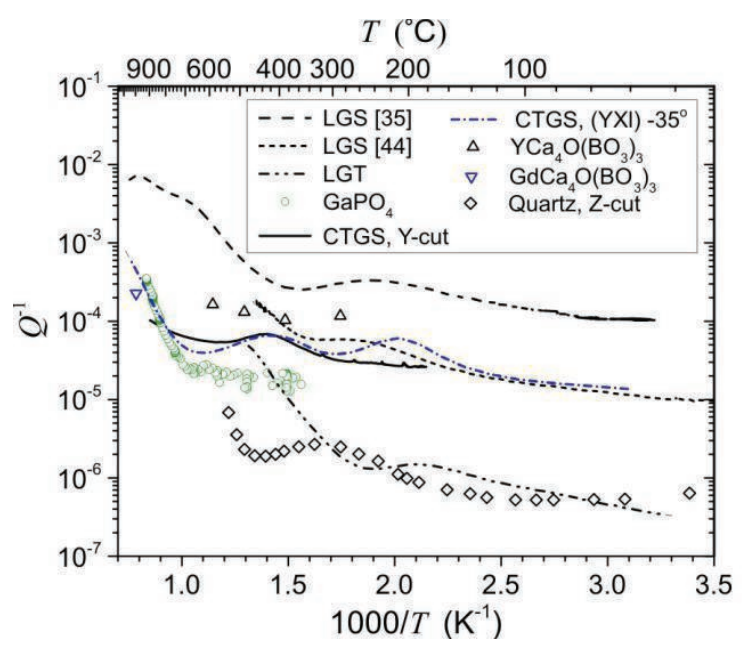

Fig. 2. Temperature dependence of acoustic $Q^{-1}$ of Y-cut LGS [37,46], Y-cut LGT [46], Y-cut $\mathrm{GaPO}_{4}$ [14], Y-cut CTGS [37], (YXI) $-35^{\circ}$ cut CTGS [37], (YXt) $-30^{\circ}$ cut $\mathrm{YCa}_{4} \mathrm{O}\left(\mathrm{BO}_{3}\right)_{3}$ [49], (YXt) $-33^{\circ}$ cut $\mathrm{GdCa} \mathrm{O}_{4} \mathrm{O}\left(\mathrm{BO}_{3}\right)_{3}$ [36], and Z-cut quartz [50] at frequencies in the range of $5 \mathrm{MHz}$ to $6 \mathrm{MHz}$.

Acoustic loss at intermediate temperatures has been reported to include contributions from several physical mechanisms. In LGS-type materials, these contributions are found to include point defect relaxations, in addition to intrinsic phonon-phonon loss and damping associated with the mechanical support of crystals $[37,46]$. There is also evidence for a broadly temperature dependent contribution that is hypothesized to be a distributed relaxation associated with dislocations [46].

The data on LGT and quartz shown in Fig. 2 provide benchmarks for the order of magnitude of $Q^{-1}$ that can be achieved in piezoelectric crystals at intermediate and ambient temperatures if anelastic defect concentrations are substantially reduced. The plotted values for LGT near ambient temperature have been shown to be dominated by intrinsic phononphonon loss [46], and those for quartz are also close to the classical limit of perfect quartz crystals [51]. Evidence that the achievable $Q^{-1}$ of LGS at low and intermediate temperatures is substantially less than values shown in Fig. 2 is also provided by measurements of Smythe et al. [15]. In that study, the $Q^{-1}$ of some LGS crystals was found to be equal to $\sim 2.0 \times 10^{-14} \omega$ at ambient temperature, corresponding to a 
value of $Q^{-1}$ at $5 \mathrm{MHz}$ that is more than an order of magnitude smaller than the lower of the two LGS data sets plotted in Fig. 2.

\section{Temperature Dependence of Frequencies}

For many potential applications, including mass sensing, the temperature dependence of resonant frequencies will degrade resolution, because of uncertainties in temperature measurements. Therefore, substantial research has been devoted to identifying crystal orientations that provide temperature compensation at elevated temperatures.

Singly-rotated thickness-shear cuts of LGT have been found to have turnover temperatures (where the derivative of frequency versus temperature is zero) as high as $180{ }^{\circ} \mathrm{C}$ [38]. $\mathrm{GaPO}_{4}$ is commercially available with orientations that provide turnover temperatures of $240{ }^{\circ} \mathrm{C}$ or $500{ }^{\circ} \mathrm{C}$. CTGS crystals with singly rotated cuts, similar to the orientation of AT-cut quartz, have been found to have turnover temperatures as high as $330^{\circ} \mathrm{C}$ [35]. On the other hand, thickness-shear modes of $\mathrm{YCa}_{4} \mathrm{O}\left(\mathrm{BO}_{3}\right)_{3}$ and $\mathrm{GdCa}_{4} \mathrm{O}\left(\mathrm{BO}_{3}\right)_{3}$ are reported to not have turnover temperatures above ambient temperatures $[36,49]$.

\section{Conclusion}

Research on piezoelectric single-crystal materials for high-temperature applications has become increasingly active during the past decade. This research has primarily been driven by sensing applications at temperatures that exceed the limits of quartz. In addition to ordered and partially disordered crystals in the langasite family, $\mathrm{GaPO}_{4}$ and rare-earth oxyborates have been a substantial focus of research. More recent entries in this research area are crystals with the structure of melilite.

The resolution and signal strength of acoustic sensors incorporating these piezoelectrics at high temperatures will be determined largely by electrical conductivity, associated acoustic loss, and compositional stability. The partial crystalline disorder of LGS and LGT presents a fundamental obstacle to lowering their acoustic loss at temperatures above $\sim 800^{\circ} \mathrm{C}$.

$\mathrm{GaPO}_{4}, \mathrm{CTGS}$, and $\mathrm{Ca}_{2} \mathrm{Al}_{2} \mathrm{SiO}_{7}$ are reported to have similar magnitudes and temperature dependences of conductivities up to $\sim 900{ }^{\circ} \mathrm{C}$. Rare-earth oxyborate crystals, such as $\mathrm{YCa}_{4} \mathrm{O}\left(\mathrm{BO}_{3}\right)_{3}$ and $\mathrm{GdCa}_{4} \mathrm{O}\left(\mathrm{BO}_{3}\right)_{3}$, are reported to have generally lower conductivities but higher activation energies, such that their conductivities approach those of $\mathrm{GaPO}_{4}$, CTGS, and $\mathrm{Ca}_{2} \mathrm{Al}_{2} \mathrm{SiO}_{7}$ at the highest measured temperatures.
Success in growing and characterizing crystals with lower conductivities has not, thus far, led to consistent lowering of the acoustic loss of resonators at elevated temperatures. This indicates that other sources of damping, including anelastic defect contributions and contact loss, are dominant over much (or all) of the measured temperature ranges. Therefore, progress in the development of innovative piezoelectric crystals for high-temperature sensors is anticipated to require integrated research on crystal growth, crystal electrochemistry, and physical acoustics, with a focus on minimization of anelastic defects.

\section{References}

[1] H. Fritze, Meas. Sci. Technol., vol. 22, p. 012002, 2011.

[2] M. Pereira da Cunha, R. J. Lad, T. B. Pollard, D. McCann, E. McCarthy, P. Prata and R. Kelley, Proc. 2014 IEEE Int. Ultrason. Symp., p. 381, 2014.

[3] M. Pereira da Cunha, R. Lad and P. Davulis et al., Proc. 2011 IEEE Topical Conf. Wireless Sensors and Sensor Networks, pp. 41-44, 2011.

[4] S. Z. Zhang, X. Jiang, M. Lapsley, P. Moses and T. R. Shrout, Appl. Phys. Lett., vol. 96, p. 013506, 2010.

[5] X. Jiang, K. Kim, S. Zhang, J. Johnson and G. Salazar, Sensors, vol. 14, pp. 144-169, 2014.

[6] J. Millichamp, E. Ali and N. P. Brandon et al., Industrial \& Engineering Chemistry Research, vol. 50, pp. 8371-8375, 2011.

[7] S. F. Grimshaw, Proc. SPIE, vol. 5527, pp. 98-104, 2004.

[8] J. W. Elam and M. J. Pellin, Anal. Chem., vol. 77, pp. 3531-3535, 2005.

[9] H. Habuka and Y. Tanaka, ECS Journal of Solid State Science and Technology, vol. 1, pp. P62-P65, 2012.

[10] D. Scaccabarozzi, B. Saggin and M. Tarabini et al., Adv. Space Res., vol. 54, pp. 2386-2397, 2014.

[11] H. Thanner, P. W. Krempl, R. Selic, W. Wallnofer and P. M. Worsch, J. Therm. Anal. Calorim., vol. 71, p. 53-59, 2003.

[12] W. L. Johnson and E. Mansfield, Proc. 2012 IEEE Int. Freq. Cntrl. Symp., pp. 1-5, 2012.

[13] J. Kosinski, R. Pastore, E. Bigler, M. Pereira da Cunha, D. C. Malocha and J. Detaint, Proc. 2001 IEEE Freq. Ctrl. Symp., pp. 278-286, 2001.

[14] H. Fritze, J. Electroceram., vol. 26, p. 122161, 2011. 
[15] R. C. Smythe, R. C. Helmbold, G. E. Hague and K. A. Snow, IEEE Trans. Ultrason. Ferro. Freq. Ctrl., vol. 47, pp. 355-3600, 2000.

[16] B. Chai, J. Lefaucheur, Y. Ji and H. Qiu, 1998 IEEE Int. Freq. Contrl. Symp., p. 748760, 1998.

[17] B. V. Mill and Y. V. Pisarevsky, Proc. 2000 IEEE/EIA Int. Freq. Ctrl. Symp., p. 133, 2000.

[18] M. M. C. Chou, S. Jen and B. H. T. Chai, Proc. 2001 IEEE Ultrason. Symp., p. 225, 2001.

[19] S. Zhang, Y. Zheng, H. Kong, J. Xin, E. Frantz and T. R. Shrout, J. Appl. Phys., vol. 105, p. 114107, 2009.

[20] K. Xiong, Y. Zheng, X. Tu and S. Zhang, J. Cryst. Growth, vol. 401, pp. 820-823, 2014.

[21] A. Zarka, B. Capelle, J. Detaint, D. Palmier, E. Philippot and O. V. Zvereva, Proc. 10th Euro. Freq. Time Forum, p. 308-312, 1996.

[22] J. Nosek, IEEE T. Ultrason. Ferr., vol. 50, pp. 571-576, 2003.

[23] J. Haines, O. Cambon, N. Prudhomme, G. Fraysse, D. Keen, L. Chapon and M. Tucker, Phys. Rev. B, vol. 73, p. 014103, 2006.

[24] F. Yu, S. Hou, X. Zhao and S. Zhang, IEEE Trans. Ultrason. Ferr., vol. 61, p. 13441356, 2014.

[25] Y. Y. Zhang, X. Yin, H. H. Yu, H. J. Cong, H. J. Zhang, J. Y. Wang and R. I. Boughton, Cryst. Growth Des., vol. 12, p. 622-628, 2012.

[26] M. Hagiwara, H. Noguchi, T. Hoshina and H. Takeda, Jpn. J. Appl. Phys., vol. 52, 2013.

[27] H. Takeda, H. Manabu, N. Hiroaki, H. Takuya, T. Tomoko, K. Nobuhiro and T. Takaaki, Appl. Phys. Lett., vol. 102, p. 242907, 2013.

[28] C. Shen, H. Zhang, Y. Zhang, J. Wang, S. Zhang and T. R. Shrout, 2014 IEEE Int. Ferro., 2014.

[29] M. Yamaga, Y. Tanii, N. Kodama, T. Takahashi and M. Honda, Phys. Rev. B, vol. 65, p. 235108, 2002.

[30] K. Shimamura, H. Takeda, T. Kohno and T. Fukuda, J. Cryst. Growth, vol. 163, pp. 388-392, 1996.

[31] K. Jacobs, P. Hofmann, D. Klimm, J. Reichow and M. Schneider, J. Solid State Chem., vol. 149, p. 180-188, 2000.

[32] W. G. Cady, Piezoelectricity, Vols. 1 and 2, New York: Dover Publications, 1964.
[33] A. Kaminskii, Phys. Status Solidi A, vol. 80, pp. 607-620, 1983.

[34] M. Souleiman, G. M. Bhalerao, T. Guillet, A. Haidoux, M. Cambon, C. Levelut, J. Haines and O. Cambon, J. Cryst. Growth, vol. 397, p. 29-38, 2014.

[35] F. Yu, S. Zhang, X. Zhao, D. Yuan, L. Qin, Q.-M. Wang and T. R. Shrout, J. Appl. Phys., vol. 109, p. 114103, 2011.

[36] S. Zhang, E. Frantz, R. Xia, W. Everson, J. Randi, D. W. Snyder and a. T. R. Shrout, J. Appl. Phys., vol. 104, p. 084103, 2008.

[37] W. L. Johnson, M. Schulz and H. Fritze, IEEE T. Ultrason. Ferr., vol. 61, p. 1433, 2014.

[38] F. Yu, X. Zhao, L. Pan, F. Li, D. Yuan1 and a. S. Zhang, J. Phys. D Appl. Phys., vol. 43, p. 165402, 2010.

[39] S. Zhang, F. Yu, R. Xia, Y. Fei, E. Frantz, X. Zhao, D. Yuan, B. H. Chai, D. Snyderd and a. T. R. Shrout, J. Cryst. Growth, vol. 318, pp. 884-889, 2011.

[40] H. Seh and H. L. Tuller, J. Electroceram., vol. 15, p. 193-202, 2005.

[41] H. Seh and H. L. Tuller, J. Electroceram., vol. 16, p. 115-125, 2006.

[42] H. Fritze, M. Schulz, H. Seh, H. L. Tuller, S. Ganschow and K. Jacobs, Solid State lonics, vol. 177, p. 3171-3174, 2006.

[43] H. Fritze, M. Schulz, H. Seh and H. L. Tuller, Mater. Res. Soc. Symp. Proc., vol. 828, pp. 145 - 150, 2005.

[44] H. Fritze, O. Schneider, H. Seh, H. L. Tuller and a. G. Borchardt, Phys. Chem. Chem. Phys., vol. 5, p. 5207-5214, 2003.

[45] A. R. Hutson and D. L. White, J. Appl. Phys, vol. 33, p. 40, 1962.

[46] W. L. Johnson, S. A. Kim, S. Uda and C. F. Rivenbark, J. Appl. Phys., vol. 110, p. 123528, 2011.

[47] A. S. Nowick and B. S. Berry, Anelastic Relaxation in Crystalline Solids, New York: Academic, 1972.

[48] H. Seh, H. Fritze and H. L. Tuller, J. Electroceram., vol. 18, pp. 139-147, 2007.

[49] H. Zu, H. Wu, Y. Wanga, S. Zhang, T. R. Shrout and Q.-M. Wanga, Sensor. Actuat. A-Phys., vol. 216, pp. 167-175, 2014.

[50] D. B. Fraser, "Impurities and Anelasticity in Crystalline Quartz," in Physical Acoustics, Vol. V, New York, Academic Press, 1968, pp. 59-110.

[51] A. W. Warner, Bell Sys. Tech. J., vol. 39, pp. 1193-1217, 1960. 